QUARTERLY OF APPLIED MATHEMATICS

VOLUME LXIV, NUMBER 3

SEPTEMBER 2006, PAGES 547-560

$\mathrm{S}$ 0033-569X(06)01025-2

Article electronically published on July 5, 2006

\title{
SPATIAL BEHAVIOR FOR A FOURTH-ORDER DISPERSIVE EQUATION
}

\author{
BY \\ RAMOn QUintanilla (Matemática Aplicada 2 Universidad Politècnica de Catalunya Colón, 11, \\ Terrassa. Barcelona, Spain) \\ AND \\ GIUSEPPE SACCOMANDI (Dipartimento di Ingegneria dell'Innovazione, Sezione Industriale, \\ Università di Lecce, Italy)
}

\begin{abstract}
In this note we investigate the spatial behavior of a linear equation of fourth order which models several mechanical situations when dispersive and dissipative effects are taken into account. In particular, this equation models the extensional vibration of a bar when we assume that external friction, with a rough substrate for example, is present. We show that for such an equation a Phragmén-Lindelöf alternative of exponential type can be obtained. A bound for the amplitude term in terms of boundary data is obtained. Moreover, when friction is absent, we obtain exponential decay results in the case of harmonic vibrations and we prove a polynomial decay estimate for general solutions.
\end{abstract}

1. Introduction. In recent years much attention has been directed to the study of the damping of end effects in several thermomechanical situations and to the general study of the spatial behavior of solutions of partial differential equations and systems. The history and development of these questions was extensively surveyed by Horgan and Knowles [11] in a work periodically updated by Horgan [9, 10. Moreover, the books by Ames and Straughan [1, Flavin and Rionero [6] and Antontsev et al. 2] are devoted to such matters. The energy method is widely used to study the spatial behavior of solutions of partial differential systems.

In this note we investigate the spatial behavior of a linear equation of fourth order which is encountered in several frameworks when we take into account dispersive effects.

Received December 14, 2005.

2000 Mathematics Subject Classification. Primary 35Q72; Secondary 74J05.

Key words and phrases. Phragmén-Lindelöf alternative, dispersive effects, friction.

This work is supported by the project "Aspectos de Estabilidad en Termomecánica"(BFM2003-00309), by the GNMF of INDAM and PRIN 2003 "Problemi Matematici Nonlineari e Stabilità nei Modelli del Continuo". GS acknowledges the hospitality and support of UPC..

E-mail address: Ramon.Quintanilla@upc.edu

E-mail address: giuseppe.saccomandi@unile.it 
In this equation the fourth-order derivative is a mixed one: second order in space and second order in time. Because we are interested in understanding the interplay between dissipation and dispersion our equation contains a first-order derivative of the displacement with respect to the time with the aim to model a source of external friction. The case where dissipation is due to the inherent constitutive properties of the material (as in viscoelasticity) has been recently considered by Leseduarte and Quintanilla [14.

In the mechanics of solids the fourth-order equation considered here, in the onedimensional and purely dispersive case, was first derived by Chree in the study of the longitudinal vibration of a bar ([15, p.428). In this framework the dispersive term arises to take into account the lateral inertia of the bar, and the dissipative first-order term may be introduced to model the contact of the bar with a rough substrate or a viscous external medium (see [21]).

A similar fourth-order equation, but containing a dissipative third-order derivative, was derived in the framework of the Mooney-Rivlin viscoelastic solids of second grade by Hayes and Saccomandi [7] in studying the propagation of transverse homogeneous waves. It is interesting to point out that Destrade and Saccomandi [3] recently discovered that viscoelastic solids of second grade contain the phenomenological theory of a continuous body with an intrinsic length proposed by Rosenau, Rubin and Gottlieb [19]. Therefore, if we consider in the framework of the theory of Rosenau, Rubin and Gottlieb a linear elastic medium with an external source of friction, we recover exactly the equation studied here. The presence of a fourth-order mixed derivative is in contrast to other nonlocal theories of solids (as second gradient elasticity), where we have purely spatial higher-order derivatives.

In fluid mechanics a fourth-order equation similar to the one studied here may be derived in the acoustic limit of the van Wijngaarden theory of bubbly liquids [22, the linearized theory of micro-stretch fluids [4] and the linear limit of the Marshall and Naghdi theory of turbulence as recently pointed out by Holm and coworkers 8 .

In some sense we are considering the linear counterpart of the so-called good Boussinesq equation that is not a completely integrable equation as the usual Boussinesq equation, but has many nice qualitative properties. About this point we refer to the detailed discussion in [16. What is striking about such higher-order differential equations is that, as far as we know, only little is known with respect to any kind of explicit solution of boundary value problems beyond classical existence results. Indeed, even though the Cauchy and backward-Cauchy problem in the one-dimensional case for such equations were studied in detail by Saxton in 1982 [20, the solution for the classical Stokes problem was provided, when internal friction is present, only recently [12. In the purely dispersive case (no dissipation) Destrade and Saccomandi provided some simple exact solutions in closed form in [3].

For all these reasons we think that it is of interest to carry out a detailed study of standard spatial estimates. In such a way we hope to improve our understanding of the various models mentioned above and to have a framework of easy comparison with the other theories of dispersive phenomena given in the literature. Moreover, it is of special interest to understand the interplay between dissipation and dispersion and their deep connection in view of what has been shown recently in 3 . 
The plan of the paper is the following. Section 2 is devoted to formulation of the basic equations of the problem. Section 3 is devoted to obtaining the spatial-growth estimates in the case where friction is present; we show that a Phragmén-Lindelöf alternative can be obtained. An estimate for the amplitude term by means of the boundary conditions is given in Section 4. These results cannot be extended to the purely dispersive case when friction is absent. To study the purely dispersive case in Section 5 we study the spatial behavior for the amplitude term when we restrict our interest to harmonic vibrations. Motivated by the results obtained in the framework of harmonic vibrations in Section 6 we prove the polynomial decay of general solutions in the case where friction is absent. It is worth noting that to prove this polynomial decay we use an argument which is usual in the studies of the time decay of solutions (see for instance [17]), but as far as we know it has never been used in the spatial estimates.

In this article we use the summation and differentiation conventions. Summation over repeated indices is assumed and differentiation in the direction $x_{k}$ is denoted by,$k$.

2. Basic equations. It is well known that a general simple continuum is characterized by the response functions $\{\psi, \eta, \varepsilon, \mathbf{T}, \xi, \mathbf{p}\}$, where, as usual, $\psi$ is the specific (per unit mass) Helmholtz free energy, $\eta$ is the specific entropy, $\varepsilon$ is the specific internal energy, $\mathbf{T}$ is the Cauchy stress, $\xi$ is the part of the specific rate of internal production of entropy due to material dissipation, and $\mathbf{p}$ is the entropy flux per unit present area. Following [19] we consider an elastic continuum $\left\{\psi_{1}, \eta_{1}, \varepsilon_{1}, \mathbf{T}_{1}, \xi_{1}, \mathbf{p}_{1}\right\}$ modified to include an intrinsic length scale by adding terms such that

$$
\psi=\psi_{1}+\psi_{2}, \quad \varepsilon=\varepsilon_{1}+\psi_{2}, \quad \mathbf{T}=\mathbf{T}_{1}+\mathbf{T}_{2},
$$

and the other quantities are not modified.

In (2.1) the functions $\psi_{2}$ and $\mathbf{T}_{2}$ are determined so that the additive terms satisfy the balance of angular momentum and cause no material dissipation, i.e.,

$$
\mathbf{T}_{2}^{T}=\mathbf{T}_{2}, \quad \rho \frac{d \psi_{2}}{d t}=\mathbf{T}_{2} \cdot \mathbf{D}
$$

where $\rho$ is the current mass density, $\mathbf{D}$ is the symmetric part of the velocity gradient, $\mathbf{L}=\operatorname{grad}(\mathbf{v})$ and the dot, $\cdot$, is the usual inner product between second-order tensors. In the simplest case [19] proposes that

$$
\mathbf{T}_{2}=\rho \alpha^{2}\left[\operatorname{grad}(\mathbf{a})+\operatorname{grad}(\mathbf{a})^{T}+2 \mathbf{L}^{T} \mathbf{L}-4 \mathbf{D}^{2}\right], \quad \psi_{2}=\alpha^{2} \mathbf{D} \cdot \mathbf{D},
$$

where $\mathbf{a}$ is the acceleration and $\alpha$ is a material constant that determines the intrinsic length.

In the case of a linear elastic and isotropic material, linearizing the quantities in (2.3) as well, we obtain for the infinitesimal displacement, $\mathbf{u}=\mathbf{u}(\mathbf{x}, t)$, the vectorial equation

$$
(\lambda+\mu) \operatorname{grad} \operatorname{div} \mathbf{u}+\mu \triangle \mathbf{u}+\alpha^{2} \triangle \mathbf{u}_{, t t}+\rho_{0} \mathbf{b}=\rho_{0} \mathbf{u}_{, t t},
$$

where $\lambda$ and $\mu$ are the material moduli, $\rho_{0}$ is the density and $\mathbf{b}$ is the body force.

In this paper we are interested in a scalar version of the system (2.4) where we assume that the body force $\mathbf{b}$ is of a dissipative kind, i.e., is linearly proportional to $\mathbf{u}_{, t}$. Therefore 
we will study the equation

$$
u_{, t t}-\epsilon \triangle u_{, t t}+b u_{, t}=a \triangle u
$$

where $\epsilon, a$ and $b$ are positive constants obtained by a suitable rescaling of the corresponding constants in (2.4). The spatial one-dimensional version of the scalar equation (2.5) may be obtained from the system (2.4) if we consider a single longitudinal wave. The two-dimensional version appears if we consider anti-plane wave motion. Finally the three-dimensional case appears if we restrict our attention to the dilatational wave associated with the Lamé potential representation of the displacement field.

It is worth noting that the time asymptotic behavior of this equation was recently studied in [18. We also note that this equation is different from the one considered in the acoustic limit of the theory of bubbly liquids developed in [4] and recently studied in 14. or in the study of transverse waves in Mooney-Rivlin materials, because of the appearance of the dissipative term $u_{, t}$ instead of $\Delta u_{, t}$.

We also note that in the absence of dissipative body force the scalar equation (2.5) becomes

$$
u_{, t t}-\epsilon \triangle u_{, t t}=a \triangle u \text {. }
$$

This equation will also be studied in this paper.

Let $R$ be the cylinder $(0, \infty) \times D$, where $D$ is a two-dimensional bounded domain such that the boundary $\partial D$ is smooth enough to apply the divergence theorem. Let $D(z)$ be the cross section of the points in $R$ such that $x_{1}=z$, and let $R(z)$ be the points of the cylinder such that $x_{1}>z$. By $R\left(z_{0}, z\right)$, we denote the points in $R$ such that the first component is greater than $z_{0}$ and lower than $z$. The equations we study here are defined on the semi-infinite cylinder $R$ and $u$ is constrained to be zero on the lateral sides of the cylinder. Thus, we add to equation (2.5) the following conditions:

$$
u=0, \quad \mathbf{x} \in[0, \infty) \times \partial D .
$$

Moreover, we impose boundary conditions on the finite end of the cylinder. Thus, we take as assumptions

$$
u\left(0, x_{2}, x_{3}, t\right)=f\left(x_{2}, x_{3}, t\right), \quad\left(x_{2}, x_{3}\right) \in D .
$$

To have a well-determined problem we need to impose initial conditions. Here, we assume null initial conditions. Thus

$$
u\left(x_{i}, 0\right)=u_{, t}\left(x_{i}, 0\right)=0 .
$$

As will be seen in the analysis, the estimates we will obtain also work for the onedimensional case.

3. Phragmén-Lindelöf alternative. In this section we study the spatial asymptotic behavior of the solutions of the problem determined by equation (2.5), boundary conditions (2.7), (2.8), and initial conditions (2.9).

To make our analysis easier we note that the following relation

$$
\left(u_{, t}\left[u_{, i}+\frac{\epsilon b}{a} u_{, t i}\right]\right)_{, i}=\frac{1}{2} \frac{d}{d t}\left(|\nabla u|^{2}+a^{-1}\left(u_{, t}-\epsilon \triangle u_{, t}\right)^{2}+\epsilon(\triangle u)^{2}\right)+\frac{b}{a} u_{, t}^{2}+\frac{\epsilon b}{a}\left|\nabla u_{, t}\right|^{2}
$$


is satisfied for every solution of the equation (2.5). In this case the analysis starts by considering the function

$$
\Phi(z, t)=-\int_{0}^{t} \int_{D(z)} \exp (-\omega s)\left(u_{, s}\left(u_{, 1}+\frac{\epsilon b}{a} u_{, s 1}\right)\right) d a d s .
$$

Using the divergence theorem and the initial and boundary conditions from (3.2) we obtain

$$
\begin{gathered}
\Phi(z, t)=\Phi\left(z_{0}, t\right)-\int_{0}^{t} \int_{R\left(z_{0}, z\right)} \exp (-\omega s)\left(\frac{\omega}{2}\left[|\nabla u|^{2}+a^{-1}\left(u_{, s}-\epsilon \triangle u, s\right)^{2}+\epsilon(\triangle u)^{2}\right]\right. \\
\left.+\frac{b}{a} u_{, s}^{2}+\frac{\epsilon b}{a}\left|\nabla u_{, s}\right|^{2}\right) d v d s \\
-\frac{1}{2} \int_{R\left(z_{0}, z\right)} \exp (-\omega t)\left(|\nabla u|^{2}+a^{-1}\left(u_{, t}-\epsilon \triangle u_{, t}\right)^{2}+\epsilon(\triangle u)^{2}\right) d v, \quad z \geq z_{0} \geq 0 .
\end{gathered}
$$

If $\Phi(z, t) \rightarrow 0$ as $z \rightarrow \infty$, then

$$
\begin{gathered}
\Phi(z, t)=\frac{1}{2} \int_{R(z)} \exp (-\omega t)\left[|\nabla u|^{2}+a^{-1}\left(u_{, t}-\epsilon \triangle u, t\right)^{2}+\epsilon(\triangle u)^{2}\right] d v \\
+\int_{0}^{t} \int_{R(z)} \exp (-\omega s)\left(\frac{\omega}{2}\left[|\nabla u|^{2}+a^{-1}\left(u_{, s}-\epsilon \triangle u_{, s}\right)^{2}+\epsilon(\triangle u)^{2}\right]\right. \\
\left.+\frac{b}{a} u_{, s}^{2}+\frac{\epsilon b}{a}\left|\nabla u_{, s}\right|^{2}\right) d v d s .
\end{gathered}
$$

Moreover, by a simple differentiation,

$$
\begin{gathered}
\frac{\partial \Phi}{\partial z}(z, t)=-\frac{1}{2} \int_{D(z)} \exp (-\omega t)\left[|\nabla u|^{2}+a^{-1}\left(u_{, t}-\epsilon \triangle u_{, t}\right)^{2}+\epsilon(\triangle u)^{2}\right] d a \\
-\int_{0}^{t} \int_{D(z)} \exp (-\omega s)\left(\frac{\omega}{2}\left[|\nabla u|^{2}+a^{-1}\left(u_{, s}-\epsilon \triangle u_{, s}\right)^{2}+\epsilon(\triangle u)^{2}\right]\right. \\
\left.+\frac{b}{a} u_{, s}^{2}+\frac{\epsilon b}{a}\left|\nabla u_{, s}\right|^{2}\right) d a d s .
\end{gathered}
$$

The next step is to estimate the absolute value of $\Phi(z, t)$ in terms of its spatial derivative to obtain an inequality of the type

$$
|\Phi(z, t)| \leq-\lambda_{\omega} \frac{\partial \Phi}{\partial z} .
$$

Using the arithmetic-geometric mean inequality (see [6, p. 335) it is possible to obtain

$$
|\Phi| \leq \int_{0}^{t} \exp (-\omega s)\left(\frac{\epsilon_{1}+\epsilon_{2}}{2} \int_{D} u_{, s}^{2} d a+\frac{\epsilon b}{2 a \epsilon_{1}} \int_{D}\left|\nabla u_{, s}\right|^{2} d a+\frac{b}{2 \epsilon_{2}} \int_{D}|\nabla u|^{2} d a\right) d s,
$$

where $\epsilon_{1}$ and $\epsilon_{2}$ are arbitrary positive constants, and taking

$$
\lambda_{\omega}=\frac{1}{2} \sqrt{\frac{(\omega+2 b) a}{\omega b}},
$$

we arrive at the following result:

$$
|\Phi| \leq \lambda_{\omega} \int_{0}^{t} \exp (-\omega s)\left(\frac{b}{a} \int_{D} u_{, s}^{2} d a+\frac{\epsilon b}{a} \int_{D}\left|\nabla u_{, s}\right|^{2} d a+\frac{\omega}{2} \int_{D}|\nabla u|^{2} d a\right) d s .
$$

Introducing (3.5) into (3.9) it is possible to obtain the estimate (3.6). 
Inequalities such as (3.6) are well known and often used in the study of spatial decay estimates for partial differential equations. This is because (3.6) implies that

$$
\Phi \leq-\lambda_{\omega} \frac{\partial \Phi}{\partial z} \text { and }-\Phi \leq-\lambda_{\omega} \frac{\partial \Phi}{\partial z}
$$

and from these statements we obtain an alternative of Phragmén-Lindelöf type which states (see [5]) that the solutions either grow exponentially for $z$ sufficiently large with the measure

$$
\begin{aligned}
& \int_{0}^{t} \int_{R(0, z)} \exp (-\omega s)\left(\frac{\omega}{2}\left[|\nabla u|^{2}+a^{-1}\left(u_{, s}-\epsilon \triangle u_{, s}\right)^{2}+\epsilon(\triangle u)^{2}\right]+\frac{b}{a} u_{, s}^{2}+\frac{\epsilon b}{a}\left|\nabla u_{, s}\right|^{2}\right) d v d s \\
& +\frac{1}{2} \int_{R(0, z)} \exp (-\omega t)\left(|\nabla u|^{2}+a^{-1}\left(u_{, t}-\epsilon \triangle u_{, t}\right)^{2}+\epsilon(\triangle u)^{2}\right) d v, \quad z \geq 0,
\end{aligned}
$$

or decay exponentially in the form

$$
\mathcal{E}_{\omega}(z, t) \leq \mathcal{E}_{\omega}(0, t) \exp \left(-\lambda_{\omega}^{-1} z\right)
$$

for all $z \geq 0$, where

$$
\begin{gathered}
\mathcal{E}_{\omega}(z, t)=\frac{1}{2} \int_{R(z)} \exp (-\omega t)\left(|\nabla u|^{2}+a^{-1}\left(u, t-\epsilon \triangle u_{, t}\right)^{2}+\epsilon(\triangle u)^{2}\right) d v \\
+\int_{0}^{t} \int_{R(z)} \exp (-\omega s)\left(\frac{\omega}{2}\left[|\nabla u|^{2}+a^{-1}\left(u_{, s}-\epsilon \triangle u_{, s}\right)^{2}+\epsilon(\triangle u)^{2}\right]\right. \\
\left.+\frac{b}{a} u_{, s}^{2}+\frac{\epsilon b}{a}\left|\nabla u_{, s}\right|^{2}\right) d v d s .
\end{gathered}
$$

In summary the estimates just derived allow us to state the following result:

TheORem 3.1. Let $u$ be a solution of the initial-boundary value problem determined by (2.5), (2.7)-(2.9). Then, either the function $\Phi(z, t)$ becomes exponentially unbounded when $z$ goes to infinity or it satisfies the spatial decay estimate (3.11).

As a corollary of this theorem, using (3.11) and the definition in (3.12), we see that for every solution which does not become unbounded for $z \rightarrow \infty$, the following estimate must hold:

$$
\mathcal{E}_{0}(z, t) \leq \exp \left(\omega t-\lambda_{\omega}^{-1} z\right) \mathcal{E}_{\omega}(0, t) .
$$

It is important to emphasize that the Phragmén-Lindelöf result here obtained is quite different from the one usually obtained in the framework of the classical hyperbolic problems (i.e., when dispersive effects are not taken into account). Moreover, our analysis does not work in the limit $b \rightarrow 0$ (i.e., no friction) and this is because $\lambda_{\omega}$ in (3.8) blows up. Therefore, to the best of our knowledge, the study of the spatial behavior of solutions of equation (2.5) in the purely dispersive case when friction or dissipative terms are neglected is an (interesting) open question. Indeed, it is well known that in the nonlinear case pure dispersion is sufficient to balance nonlinearity to avoid shock wave formation. Therefore it seems reasonable to conjecture that in the linear case the presence of dispersion would be sufficient to allow some interesting estimates and allow an asymptotic behavior in time of the solutions also when dissipation is not considered as well. 
We note that in the other degenerate case $\omega=0$, it is possible to obtain an alternative decay estimate from (3.11). To this end we define

$$
F(z, t)=-\int_{0}^{t} \int_{D(z)} u_{, s}\left(u_{, 1}+\frac{\epsilon b}{a} u_{, 1 s}\right) d a d s
$$

From (3.7) and the Poincaré-type inequality (see [6], p.338)

$$
\int_{0}^{t} h^{2} d s \leq \frac{4 t^{2}}{\pi^{2}} \int_{0}^{t}\left[h^{\prime}(s)\right]^{2} d s
$$

which is satisfied whenever $h(0)=0$, we obtain

$$
|F| \leq\left[\frac{2\left(\epsilon_{1}+\epsilon_{2}\right) s^{2}}{\pi^{2}}+\frac{\epsilon b}{2 a \epsilon_{1}}\right] \int_{0}^{t} \int_{D}|\nabla u, s|^{2} d a d s+\frac{b}{2 \epsilon_{2}} \int_{0}^{t} \int_{D}|\nabla u|^{2} d a d s
$$

Here $\epsilon_{1}$ and $\epsilon_{2}$ are two arbitrary positive constants which can be selected to obtain a bound. To recover an estimate similar to (3.6) for the function $F$, we need introduce a function depending on time $t$ instead of the parameter $\lambda_{0}$.

Obviously, the analysis of this section also applies to the relevant one-dimensional case where our equation (2.5) reduces to the equation for the longitudinal vibrations of a rod [15] introduced by Pochamner and Chree.

4. The amplitude term. To have a more detailed knowledge and understanding of the decay estimates here proposed, we give an estimate for the term $\mathcal{E}_{\omega}(0, t)$, which is the amplitude term.

Let us assume that $u\left(x_{i}, t\right)$ is a solution of the initial-boundary value problem satisfying the decay estimate (3.11). Let $\xi\left(x_{i}, t\right)$ be a regular function which satisfies the boundary conditions and tends to zero uniformly (in $x_{2}, x_{3}$ and $t$ ) when $z$ goes to infinity. We know that

$$
\Phi(0, t)=-\int_{0}^{t} \int_{D(0)} \exp (-\omega s)\left(\xi, s\left[u_{, 1}+\frac{\epsilon b}{a} u_{, 1 s}\right]\right) d a d s
$$

It follows that

$$
\begin{aligned}
\Phi(0, t) & =\int_{0}^{t} \int_{R} \exp (-\omega s)\left(\xi_{, i s} u_{, i}+\frac{\epsilon b}{a} \xi_{, i s} u_{, i s}+\xi_{, s} \triangle u+\frac{\epsilon b}{a} \xi_{, s} \triangle u_{, s}\right) d v d s \\
& =\frac{\epsilon b}{a} \exp (-\omega t) \int_{R} \xi_{, t} \triangle u d v \\
& +\int_{0}^{t} \int_{R} \exp (-\omega s)\left[\xi_{, i s} u_{, i}+\frac{\epsilon b}{a} \xi_{, i s} u_{, i s}+\left(1+\frac{\epsilon b \omega}{a}\right) \xi_{, s} \triangle u-\frac{\epsilon b}{a} \xi_{, s s} \triangle u\right] d v d s .
\end{aligned}
$$


Using the Hölder inequality (see [6], p. 335) we obtain

$$
\begin{aligned}
\Phi(0, t) \leq & \exp (-\omega t) \frac{\epsilon b}{a}\left(\int_{R} \xi_{, t}^{2} d v\right)^{1 / 2}\left(\int_{R}(\triangle u)^{2} d v\right)^{1 / 2} \\
& +\left(\int_{0}^{t} \int_{R} \exp (-\omega s)\left[\frac{a+\epsilon b}{a}\left|\nabla \xi_{, s}\right|^{2}+\left|\frac{a+\epsilon b \omega}{a}\right| \xi_{, s}^{2}+\frac{\epsilon b}{a} \xi_{, s s}^{2}\right] d v d s\right)^{1 / 2} \\
& \times\left(\int_{0}^{t} \int_{R} \exp (-\omega s)\left[|\nabla u|^{2}+\frac{\epsilon b}{a}|\nabla u, s|^{2}+\frac{a+\epsilon b \omega}{a}(\triangle u)^{2}+\frac{\epsilon b}{a}(\triangle u)^{2}\right] d v d s\right)^{1 / 2}
\end{aligned}
$$

From (4.2) and (4.3) it follows that

$$
\begin{aligned}
\Phi(0, t) & \leq \Phi(0, t)^{1 / 2}\left[\exp \left(-\frac{\omega t}{2}\right) \frac{\epsilon^{1 / 2} b}{a}\left(\int_{R} \xi_{, t}^{2} d v\right)^{1 / 2}\right. \\
& \left.+K\left(\int_{0}^{t} \int_{R} \exp (-\omega s)\left(\frac{a+\epsilon b}{b}\left|\nabla \xi_{, s}\right|^{2}+\frac{a+\epsilon b \omega}{a} \xi_{, s}^{2}+\frac{\epsilon b}{a} \xi_{, s s}^{2}\right) d v d s\right)^{1 / 2}\right]
\end{aligned}
$$

where

$$
K^{2}=\max \left\{\frac{2}{\omega}, 1, \frac{|2(a+\epsilon b \omega+\epsilon b)|}{\omega a \epsilon}\right\}
$$

This means that

$$
\Phi(0, t) \leq 2\left(\frac{\epsilon b^{2}}{a^{2}} \exp (-\omega t) A^{*}+K^{2} B\right)
$$

where

$$
A^{*}=\int_{R} \xi_{, t}^{2} d v
$$

and

$$
B=\int_{0}^{t} \int_{R} \exp (-\omega s)\left(\frac{a+\epsilon b}{a}\left|\nabla \xi_{, s}\right|^{2}+\frac{a+\epsilon b \omega}{a} \xi_{, s}^{2}+\frac{\epsilon b}{a} \xi_{, s s}^{2}\right) d v d s .
$$

Let us take the function

$$
\xi\left(x_{i}, t\right)=f\left(x_{2}, x_{3}, t\right) \exp \left(-\nu x_{1}\right),
$$

where $\nu$ is an arbitrary positive constant. By simple computations we may derive that

$$
\begin{aligned}
\xi_{, t} & =f_{, t}\left(x_{2}, x_{3}, t\right) \exp \left(-\nu x_{1}\right), & \xi_{, t t} & =f_{, t t}\left(x_{2}, x_{3}, t\right) \exp \left(-\nu x_{1}\right), \\
\xi_{, 1 t} & =-\nu f_{, t}\left(x_{2}, x_{3}, t\right) \exp \left(-\nu x_{1}\right), & \xi_{, \beta t} & =f_{, \beta t}\left(x_{2}, x_{3}, t\right) \exp \left(-\nu x_{1}\right),
\end{aligned}
$$

where $\beta=2,3$ and

$$
\begin{gathered}
\xi_{, t}^{2}=f_{, t}^{2} \exp \left(-2 \nu x_{1}\right), \quad \xi_{, t t}^{2}=f_{, t t}^{2} \exp \left(-2 \nu x_{1}\right), \\
\left|\nabla \xi_{, t}\right|^{2}=\left(\nu^{2} f_{, t} f_{, t}+f_{, \beta t} f_{, \beta t}\right) \exp \left(-2 \nu x_{1}\right) .
\end{gathered}
$$

Because the $\xi$ defined in (4.9) is a regular function which as required satisfies the boundary conditions and converges to zero uniformly when $z \rightarrow \infty$ in all the remaining 
independent variables, we introduce the following notation:

$$
\begin{aligned}
B_{1} & =\int_{0}^{t} \int_{R} \exp (-\omega s)\left|\nabla \xi_{, s}\right|^{2} d v d s \\
& =\frac{1}{2 \nu} \int_{0}^{t} \int_{D} \exp (-\omega s)\left(\nu^{2} f_{, s} f_{, s}+f_{, \beta s} f_{, \beta s}\right) d a d s, \\
B_{2} & =\int_{0}^{t} \int_{R} \exp (-\omega s) \xi_{, s}^{2} d v d s=\frac{1}{2 \nu} \int_{0}^{t} \int_{D} \exp (-\omega s) f_{, s}^{2} d a d s, \\
B_{3} & =\int_{0}^{t} \int_{R} \exp (-\omega s) \xi_{, s s}^{2} d v=\frac{1}{2 \nu} \int_{0}^{t} \int_{D} \exp (-\omega s) f_{, s s}^{2} d a d s, \\
A^{*} & =\int_{R} \xi_{, t}^{2} d v=\frac{1}{2 \nu} \int_{D} f_{, t}^{2} d a,
\end{aligned}
$$

and we evaluate (4.6) as

$$
\Phi(0, t) \leq 2\left[\frac{\epsilon b^{2}}{a^{2}} \exp (-\omega t) A^{*}+K^{2}\left(\frac{a+\epsilon b}{a} B_{1}+\frac{a+\epsilon b \omega}{a} B_{2}+\frac{\epsilon b}{a} B_{3}\right)\right] .
$$

The relation (4.14) is an upper bound for the amplitude term in the estimate (3.11). We note that the estimate depends on an arbitrary positive constant $\nu$ which can be optimized to make the right-hand side of (4.14) as small as possible.

5. Harmonic vibrations. Because it seems that to obtain a spatial estimate in the case of no friction $(b=0)$ is quite hard, we shall first consider the special case of harmonic vibrations. Although this is a special case, in a linear theory it is always an important case.

To this end let us consider equation (2.5) in a cylindrical domain and let us choose boundary conditions such that, on the initial cross section they vary harmonically in time whereas on the mantle they are null. Under these assumptions it is possible to search for solutions of equation (2.6) in the form

$$
u\left(x_{i}, t\right)=\exp (i \omega t) v\left(x_{i}\right) .
$$

In this case the function $v=v\left(x_{i}\right)$ satisfies the equation

$$
\left(\epsilon \omega^{2}-a\right) v_{, i i}=\omega^{2} v
$$

subject to the boundary conditions

$$
v=0, \quad \mathbf{x} \in[0, \infty) \times \partial D,
$$

and

$$
v\left(0, x_{2}, x_{3}\right)=g\left(x_{2}, x_{3}\right), \quad\left(x_{2}, x_{3}\right) \in D .
$$

In the one-dimensional case, equation (5.2) becomes the ordinary differential equation:

$$
\left(\epsilon \omega^{2}-a\right) v, 11-\omega^{2} v=0
$$

whose general solution is given as

$$
v\left(x_{1}\right)=A_{1} \exp \left(\left|\frac{\omega}{\sqrt{\epsilon \omega^{2}-a}}\right| x_{1}\right)+A_{2} \exp \left(-\left|\frac{\omega}{\sqrt{\epsilon \omega^{2}-a}}\right| x_{1}\right)
$$

whenever $\epsilon \omega^{2}>a$. We note that whereas for the amplitude term of the one-dimensional harmonic vibrations of the wave equation the only function which tends to zero when $x_{1}$ 
goes to infinity is the null solution, here we have a nontrivial decaying solution whose rate of decay is determined by

$$
-\left|\frac{\omega}{\sqrt{\epsilon \omega^{2}-a}}\right| .
$$

When $\epsilon \omega^{2}<a$ the harmonic vibrations neither blow up nor decay because they are trapped by the microstructure.

Now let us consider a two-dimensional case where the domain is given by a semiinfinite rectangular strip $(0, \infty) \times(0, L)$. In this case our problem may be reduced to the ordinary differential equation

$$
\left(\epsilon \omega^{2}-a\right)\left(A_{, 11}-\frac{n^{2} \pi^{2}}{L^{2}} A\right)-\omega^{2} A=0
$$

by the assumption $v\left(x_{1}, x_{2}\right)=A\left(x_{1}\right) \sin \left(n \pi x_{2} / L\right)$.

In this case the rate of decay is

$$
\lambda=-\sqrt{\frac{\omega^{2}}{\epsilon \omega^{2}-a}+\frac{\pi^{2}}{L^{2}}}
$$

and also a weaker requirement than $\epsilon \omega^{2}>a$ suffices. This difference with the onedimensional case is not surprising. Boundaries introduce a new dispersive effect to add to the one inherent to the intrinsic characteristic length $\epsilon$. This is because it has been necessary in the previous sections to check that the estimate was true in the one-dimensional case as well.

Also in the general case it is possible to obtain the solution of the equation (5.2) under boundary conditions (5.3) and (5.4) by means of the usual technique of separation of variables. We note that functions of the form

$$
\Theta_{n}\left(x_{2}, x_{3}\right) \exp \left(\lambda_{n} x_{1}\right)
$$

where $\Theta_{n}\left(x_{2}, x_{3}\right)$ is an eigenfunction of the clamped membrane problem with eigenvalue $\alpha_{n}$, satisfy equation (5.10) whenever

$$
\left(\epsilon \omega^{2}-a\right)\left(\lambda_{n}^{2}-\alpha_{n}\right)=\omega^{2} .
$$

Then to obtain the rate of decay of these solutions we consider two different cases:

$$
\text { i) } \epsilon \omega^{2}>a, \text { ii) } a>\left(\epsilon+\alpha_{1}^{-1}\right) \omega^{2} \text {. }
$$

From (5.11) we have

$$
\lambda_{n}=-\sqrt{\alpha_{n}+\frac{\omega^{2}}{\epsilon \omega^{2}-a}}
$$

so the rate of decay is

$$
-\sqrt{\alpha_{1}+\frac{\omega^{2}}{\epsilon \omega^{2}-a}}
$$

Moreover, when condition (ii) holds, the solution of equation (5.2) decays with a rate that is at least

$$
-\sqrt{\alpha_{1}-\frac{\omega^{2}}{a-\epsilon \omega^{2}}} .
$$


To compare our results with the ones corresponding to the usual wave equation, we recall that for such an equation the decay of solutions is only possible if $a \alpha_{1}>\omega^{2}$, and when this condition is fulfilled the rate of decay is

$$
-\sqrt{\alpha_{1}-\frac{\omega^{2}}{a}}
$$

This is because in the wave equation the decay is induced only by the dispersive effects introduced by the boundaries, and indeed in the one-dimensional case we have that decay is not possible.

6. Polynomial decay in the no-friction case. The previous section has clearly shown that an exponential decay is possible also in the no-friction case. In this section we extend these results by showing that for a general time behavior of the solutions polynomial decay is possible. Obviously this is true whenever we assume that the solution of (2.5), with boundary conditions and initial conditions (2.7)-(2.9), decays uniformly as $x_{1}$ goes to infinity, i.e.,

$$
u_{, t}, \nabla u, u_{, t t}, \nabla u_{, t} \rightarrow 0
$$

uniformly in $x_{1}$.

First, we note that for $b=0$, equality 3.1 simplifies to

$$
\left(u_{, t} u_{, i}\right)_{, i}=\frac{1}{2} \frac{d}{d t}\left(|\nabla u|^{2}+a^{-1}\left(u_{, t}-\epsilon \triangle u_{, t}\right)^{2}+\epsilon(\triangle u)^{2}\right),
$$

for any solution of our linear problem, and the same result holds when $u$ is replaced by $u, t$ so that

$$
\left(u_{, t t} u_{, t i}\right)_{, i}=\frac{1}{2} \frac{d}{d t}\left(\left|\nabla u_{, t}\right|^{2}+a^{-1}\left(u_{, t t}-\epsilon \triangle u_{, t t}\right)^{2}+\epsilon\left(\triangle u_{, t}\right)^{2}\right) .
$$

Let us define the functions

$$
\begin{aligned}
\mathcal{E}_{1 \omega}(z, t) & =\frac{1}{2} \int_{R(z)} \exp (-\omega t)\left(|\nabla u|^{2}+a^{-1}\left(u_{, t}-\epsilon \triangle u_{, t}\right)^{2}+\epsilon(\triangle u)^{2}\right) d v \\
& +\int_{0}^{t} \int_{R(z)} \exp (-\omega s)\left(\frac{\omega}{2}\left(|\nabla u|^{2}+a^{-1}\left(u_{, s}-\epsilon \triangle u_{, s}\right)^{2}+\epsilon(\triangle u)^{2}\right)\right) d v d s
\end{aligned}
$$

and

$$
\begin{aligned}
\mathcal{E}_{2 \omega}(z, t) & =\frac{1}{2} \int_{R(z)} \exp (-\omega t)\left(\left|\nabla u_{, t}\right|^{2}+a^{-1}\left(u_{, t t}-\epsilon \triangle u_{, t t}\right)^{2}+\epsilon\left(\triangle u_{, t}\right)^{2}\right) d v \\
& +\int_{0}^{t} \int_{R(z)} \exp (-\omega s)\left(\frac{\omega}{2}\left(\left|\nabla u_{, s}\right|^{2}+a^{-1}\left(u_{, s s}-\epsilon \triangle u_{, s s}\right)^{2}+\epsilon\left(\triangle u_{, s}\right)^{2}\right)\right) d v d s
\end{aligned}
$$

Using the boundary conditions, initial conditions and asymptotic conditions, we have

$$
\mathcal{E}_{1 \omega}(z, t)=-\int_{0}^{t} \int_{D(z)} \exp (-\omega s) u_{, s} u_{, 1} d a d s
$$

and

$$
\mathcal{E}_{2 \omega}(z, t)=-\int_{0}^{t} \int_{D(z)} \exp (-\omega s) u_{, s s} u_{, s 1} d a d s
$$


Moreover, with some simple algebra, we establish

$$
\begin{aligned}
\frac{\partial \mathcal{E}_{1 \omega}(z, t)}{\partial z}= & -\frac{1}{2} \int_{D(z)} \exp (-\omega t)\left(|\nabla u|^{2}+a^{-1}\left(u_{, t}-\epsilon \triangle u_{, t}\right)^{2}+\epsilon(\triangle u)^{2}\right) d a \\
& -\int_{0}^{t} \int_{D(z)} \exp (-\omega s)\left(\frac{\omega}{2}\left[|\nabla u|^{2}+a^{-1}\left(u_{, s}-\epsilon \triangle u_{, s}\right)^{2}+\epsilon(\triangle u)^{2}\right]\right) d a d s
\end{aligned}
$$

and

$$
\begin{aligned}
\frac{\partial \mathcal{E}_{2 \omega}(z, t)}{\partial z}= & -\frac{1}{2} \int_{D(z)} \exp (-\omega t)\left(\left|\nabla u_{, t}\right|^{2}+a^{-1}\left(u_{, t t}-\epsilon \triangle u_{, t t}\right)^{2}+\epsilon\left(\triangle u_{, t}\right)^{2}\right) d a \\
& -\int_{0}^{t} \int_{D(z)} \exp (-\omega s)\left(\frac{\omega}{2}\left[\left|\nabla u_{, s}\right|^{2}+a^{-1}\left(u_{, s s}-\epsilon \triangle u_{, s s}\right)^{2}+\epsilon\left(\triangle u_{, s}\right)^{2}\right]\right) d a d s .
\end{aligned}
$$

A direct calculation shows that

$$
\frac{\partial \mathcal{E}_{1 \omega}}{\partial z}+\frac{\partial \mathcal{E}_{2 \omega}}{\partial z} \leq-\frac{\omega \alpha_{1}^{1 / 2}}{2} \mathcal{E}_{1 \omega}
$$

where $\alpha_{1}$ is the first eigenvalue of the clamped membrane problem

$$
v_{, \alpha \alpha}+\alpha v=0 \text { in } D, v=0 \text { on } \partial D .
$$

From (6.9) we obtain the estimate

$$
\mathcal{L}_{\omega}(z, t)+\frac{\omega \alpha_{1}^{1 / 2}}{2} \int_{0}^{z} \mathcal{E}_{1 \omega}(s) d s \leq \mathcal{L}_{\omega}(0, t),
$$

where

$$
\mathcal{L}_{\omega}(z, t)=\mathcal{E}_{1 \omega}(z, t)+\mathcal{E}_{2 \omega}(z, t) .
$$

On the other hand, we know that the spatial derivative of $\mathcal{E}_{1 \omega}(z, t)$ is always nonpositive and therefore

$$
\frac{\partial}{\partial z}\left(z \mathcal{E}_{1 \omega}(z, t)\right)=\mathcal{E}_{1 \omega}(z, t)+z \frac{\mathcal{E}_{1 \omega}}{\partial z} \leq \mathcal{E}_{1 \omega}(z, t) .
$$

A trivial quadrature implies that

$$
z \mathcal{E}_{1 \omega}(z, t) \leq \int_{0}^{z} \mathcal{E}_{1 \omega}(p, t) d p \leq \frac{2 \alpha^{-1 / 2}}{\omega} \mathcal{L}_{\omega}(0, t),
$$

which leads to the desired polynomial decay

$$
\mathcal{E}_{1 \omega}(z, t) \leq \frac{2 \alpha^{-1 / 2}}{z \omega} \mathcal{L}_{\omega}(0, t) .
$$

We note that an upper bound for the amplitude terms $\mathcal{L}_{\omega}(0, t)$ can be obtained in terms of the boundary conditions, and this can be done following the method proposed in Section 4. Moreover, it is obvious that our analysis applies to the one-dimensional case.

7. Concluding remarks. In the previous sections we have obtained a general exponential decay estimate for the solutions of a dispersive wave equation with friction. In the case of no friction we have been able to obtain a polynomial decay estimate. Only in the special case of harmonic vibrations have we been able to obtain a general and detailed result when friction is neglected. Our results are complimentary to the ones obtained by Leseduarte and Quintanilla [14 where the dissipation is provided by a frame indifferent third-order derivative (second order in space and first order in time). Also the exponential decay results in [14] are not valid when the dissipative effects are neglected. 
Therefore a major open problem in the study of dispersive wave equations is to give an optimal decay result when dissipation is not presented. The results that we have found in the case of harmonic waves allow us to conjecture that some improvements, with respect to the results proposed here, could be possible.

We think that the analysis we have used here may easily be extended to the more general theory of nonlocal continuous media called bi-Helmholtz type elasticity theory [13. In such a way a clearer and deeper understanding of the mathematical issues related to dispersion would be possible.

\section{REFERENCES}

[1] K.A. Ames and B. Straughan. Non-Standard and Improperly Posed Problems. Academic Press, San Diego, (1997).

[2] S.N. Antontsev, J.I. Díaz and S. Shmarev. Energy Methods for Free Boundary Problems: Applications to Nonlinear PDEs and Fluid Mechanics. Birkhäuser, Boston (2002). MR1858749 (2002i:35001)

[3] M. Destrade and G. Saccomandi. Waves and vibrations in a solid of second grade, to appear in Proceedings of WASCOM 2005, World Scientific, Singapore (2005).

[4] A. C. Eringen, Theory of thermo-microstretch fluids and bubbly liquids. International Journal of Engineering Science 28, 133-143 (1990). MR.1040726 (91a:76008)

[5] J. N. Flavin, R. J. Knops and L.E. Payne, Decay estimates for the constrained elastic cylinder of variable cross section, Quart. Appl. Math., XLVII, (1989) 325-350. MR0998106 (90g:73026)

[6] J. N. Flavin and S. Rionero. Qualitative Estimates for Partial Differential Equations: An Introduction, CRC Press, Boca Raton (1996). MR1396085 (98a:35003)

[7] M. Hayes and G. Saccomandi. Finite amplitude transverse waves in special incompressible viscoelastic solids, J. of Elasticity, 59, (2000) 213-225.

[8] D. D. Holm, V. Putkaradze. P. D. Weidman and B. A. Wingate. Boundary effects on exact solutions of the Lagrangian-averaged Navier-Stokes- $\alpha$ equations, J. of Statistical Physics, 113, (2003) 841854. MR2036873 (2004k:76058)

[9] C.O. Horgan. Recent developments concerning Saint-Venant's Principle: An update, Applied Mechanics Reviews, 42, (1989) 295-303. MR 1021553 (90i:73013)

[10] C.O. Horgan. Recent developments concerning Saint-Venant's Principle: A second update, Applied Mechanics Reviews, 49, (1996) 101-111.

[11] C.O. Horgan and J.K. Knowles. Recent developments concerning Saint-Venant's Principle, Advances in Applied Mechanics (Ed. J. W. Hutchinson and T.Y. Wu), 23, Academic Press, New York (1983) 179-269. MR0889288(88a:73009)

[12] P. M. Jordan and C. Feuillade. On the propagation of transient acoustic waves in isothermal bubbly liquids, Physics Letters A, 350 (2006) 56-62.

[13] M. Lazar, G. A. Maugin and E.C. Aifantis. On a theory of nonlocal elasticity of bi-Helmholtz type and some applications, International Journal of Solids and Structures, 43 (2006) 1404-1421. MR2200992

[14] M.C. Leseduarte and R. Quintanilla. Some qualitative properties of solutions of the system governing acoustic waves in bubbly liquids. International Journal of Engineering Science, to appear (2006).

[15] A. E. H. Love. A Treatise on the Mathematical Theory of Elasticity. Dover Publications. New York, fourth edition (1944). MR0010851 (6:79e)

[16] G. A. Maugin. Nonlinear Waves in Elastic Crystals. Oxford University Press. Oxford, (1999). MR.1772390 (2002e:74002)

[17] J. Muñoz-Rivera and R. Quintanilla. On the time polynomial decay in elastic solids with voids. Manuscript (2005).

[18] A. F. Pazoto, J. C. Vila Bravo and J. E. Muñoz-Rivera. Asymptotic stability of semigroups associated to linear weak dissipative systems, Math. Computer Modelling, 40, (2004), 387-392. MR2091069 (2005e:47105)

[19] M. B. Rubin, P. Rosenau and O. Gottlieb. Continuum model of dispersion caused by an inherent material characteristic length, J. Appl. Phys. 77, (1995) 4054-4063. 
[20] R. Saxton. The Cauchy and backward-Cauchy problem for a nonlinear hyperelastic infinite rod, Lectures Notes in Mathematics, 1032, Springer-Verlag. Berlin, (1982) 427-448. MR0742654|(85k:35215)

[21] P. Villaggio. Mathematical Models for Elastic Structures. Cambridge University Press. Cambridge, (1997). MR1486043 (99e:73064)

[22] L. van Wijngaarden. One-dimensional flow of liquids containing small gas bubbles, Ann. Rev. Fluid Mech., 4, (1972) 369-396. 